The Astrophysical Journal, Vol. 159, January 1970

(C) 1970. The University of Chicago. All rights reserved. Printed in U.S.A.

\title{
ABSOLUTE SPECTRAL ENERGY DISTRIBUTION OF QUASI-STELLAR OBJECTS FROM 0.3 TO 2.2 MICRONS*
}

\author{
J. B. OKe ANd G. Neugebauer \\ Mount Wilson and Palomar Observatories, Carnegie Institute of Washington, \\ California Institute of Technology \\ AND \\ E. E. BECKLIN \\ California Institute of Technology \\ Received 1969 June 23
}

\section{ABSTRACT}

The absolute spectral energy distribution from 0.32 to $2.2 \mu$ has been obtained for twenty-eight quasistellar sources. Photometry at $2.2 \mu$ has been obtained for an additional fifteen objects. The data show that the continua over this wavelength range generally can be described with a power-law spectrum; the index varies from -0.2 to -1.6 , with the entire range being populated. For quasi-stellar objects which are known to have large-amplitude variations in visual magnitude, the energy distributions remain sensibly unchanged during the variations. No characteristic of the energy distribution in the observed range of wavelengths which distinguishes between radio-quiet and radio-active quasi-stellar objects is found. The near constancy of the observed ratio of the line intensities of $L a$ to the $C$ IV line can be interpreted as implying an electron temperature of $20000^{\circ} \mathrm{K}$. The range of equivalent widths of both $(\mathrm{H} a+[\mathrm{N} \mathrm{II}])$ and $\mathrm{L} a$ is small. The ratio of the number of photons in the Lyman continuum with respect to the number in the $\mathrm{La}$ line is not consistent with radiative recombination from an opaque source.

\section{INTRODUCTION}

Although much information about the quasi-stellar sources has been obtained from visual spectra and from photometry in the $U, B$, and $V$ wavelength bands, it is of interest to know the absolute spectral energy distribution of these objects over a large range of wavelengths. The development, over the last several years, of very efficient photoelectric spectrum scanners and of infrared techniques for use with large telescopes has made it possible to obtain absolute spectral energy distributions, extending from the visual to the infrared wavelengths, of a significant number of quasi-stellar sources.

In this paper extensive spectral energy distributions between 0.32 and $2.2 \mu$ are presented for twenty-eight quasi-stellar objects; $2.2-\mu$ photometry is presented for an additional fifteen objects. The absolute energy distributions of most of these objects have not been measured before, although several objects which were observed previously and which are either suspected or known variables have been reobserved. In some cases red and near-infrared spectrophotometric observations have been obtained for objects for which only blue results have previously been published.

The only quasi-stellar object for which a detailed and extensive energy distribution is known is the brightest of these objects, 3C 273. The absolute spectral energy distribution of 3C 273 in the visual region has been published by Oke (1965a); a much more detailed set of measures has recently been presented by Wampler and Oke (1967). Data in the infrared beyond $1 \mu$ have been published by Johnson (1964), Low and Johnson (1965), and Low (1968).

Less extensive data have been obtained by Oke $(1966,1967)$ in the visual wavelengths for 3C 9, 3C 48, 3C 245, 3C 279, 3C 286, 3C 446, and CTA 102, and by Wampler (1967a,b,

* This work supported in part by National Aeronautics and Space Administration grant NGL 05 002-007. 
1968) for 3C 9, 3C 345, 3C 380, 3C 446, MSH 14-121, 3C 249.1, PHL 938, and PKS $2251+11$. Data at $1.6 \mu$ on twenty-four quasi-stellar objects have been listed by Low (1968).

\section{INSTRUMENTATION}

Three different instruments, each covering different spectral ranges, have been used at the Hale 200-inch telescope in obtaining the data presented here: the prime-focus scanner, a new multichannel spectrometer, and a two-beam infrared photometer.

Below $\lambda 5800$ almost all the observations were made with the prime-focus scanner; the observational techniques and reduction methods have been previously described (Oke 1965b). Observations were made with exit slits which were usually 50 or $100 \AA$, and observed wavelengths were spaced so that the whole spectrum was observed without gaps. Standard deviations were estimated by comparing results obtained on different nights; these are typically between 2 and 4 percent. The prime-focus scanner was also used for some objects to measure three or four points in the wavelength range from 5800 to $11000 \AA$. In these cases the standard deviations were calculated from the total number of counts, including the sky counts.

During the summer of 1968 , a new multichannel spectrometer for use at the Cassegrain focus of the 200-inch Hale telescope was put into operation (Oke 1969). For most of the data in this paper, the instrument was used with fifteen simultaneous channels covering the whole wavelength range from 5600 to $11000 \AA$; the bandpass in each channel was $360 \AA$. The observing techniques and reductions closely parallel those used with the prime-focus scanner. Measurements made in regions of strong absorption by oxygen or water vapor could be reduced with satisfactory accuracy except from $\lambda 9000$ to $\lambda 9700$ where the extinction is very large. Standard deviations were calculated from the total counts obtained. Two objects, PKS 0405-12 and 3C 323.1, were observed with eighteen channels of the multichannel spectrometer using both blue and red photomultipliers. The bandpasses were $40 \AA$ from $\lambda 3180$ to $\lambda 5700$ and $80 \AA$ from $\lambda 5700$ to $\lambda 8400$ in PKS $0405-12$ and twice as large for 3C 323.1.

The accuracy obtained with the multichannel instrument is so much greater than that obtained from the prime-focus scanner that observations with the latter instrument are used only where either (1) they alone exist, (2) the object is a known or suspected variable, or (3) the points are sufficiently accurate to help define emission-line intensities.

Broad-band data at 1.65 and $2.2 \mu$ were obtained with a two-beam photometer (Becklin and Neugebauer 1968) which measures the difference in the radiation from two portions of the sky separated by approximately $20^{\prime \prime}$. The radiation is detected with $\mathrm{PbS}$ photoconductive cells cooled by liquid nitrogen; interference filters define the wavelength intervals. All the observations reported here were made at the Cassegrain focus of the Hale 200-inch telescope with 7".5 apertures and covered wavelengths $2.0-2.4 \mu$ and 1.5$1.8 \mu$. Observations were carried out by setting the object in one beam with the sky in the second and then reversing the roles of the two beams. Typically, integrations were carried out in one configuration for approximately 1 minute before switching beams; the total integration time was a function of the brightness of the object but was generally less than $\frac{1}{2}$ hour. Several stars of approximately 8.0 mag were observed with both the 200-inch and the 24-inch telescope on Mount Wilson to check the linearity of the system and validity of the integration procedure. The uncertainties in the measurements were determined from the statistical fluctuations in short-term $(\sim 10 \mathrm{sec})$ samples of the data. A contribution to the error on the order of 7 percent was added quadratically to account for the uncertainty in determining the nightly sensitivity. From intercomparison of twenty-one sources which were observed more than once, the errors assigned appear reasonable.

All measurements have been reduced to absolute observed fluxes $f_{\nu}\left(\mathrm{W} \mathrm{m} \mathbf{m}^{-2} \mathbf{H z}^{-1}\right)$. The fluxes obtained in the visual and near-infrared are based on the absolute calibration 
of a Lyr given by Oke (1964) except that all fluxes below the Balmer jump are made fainter by 6 percent (Mihalas 1966). The observations at $2.2 \mu$ were placed on the $K$ magnitude scale established by Johnson (1962) by comparison with stars measured by Johnson et al. (1966); those at $1.65 \mu$ were placed on a magnitude scale with a Lyr set equal to 0.0 mag. For the infrared photometry, at least one standard in the vicinity of the quasi-stellar objects was measured before and after each observation of a quasistellar object. The absolute fluxes are based on $6.5 \times 10^{-24}$ and $1.07 \times 10^{-23} \mathrm{~W} \mathrm{~m}^{-2}$ $\mathrm{Hz}^{-1}$ as the flux received from a 0.0 -mag star at 2.2 and $1.65 \mu$, respectively.

TABLE 1

QUASI-STELLAR OBJECTS WITH EXTENSIVE SPECTROPHOTOMETRIC DATA

\begin{tabular}{|c|c|c|c|}
\hline Object & $z^{*}$ & $a$ & $\begin{array}{c}\log F_{\nu_{0}}\left(10^{16} \mathrm{~Hz}\right) \\
\left(\mathrm{W} \mathrm{m}^{-2} \mathrm{~Hz}^{-1}\right)\end{array}$ \\
\hline 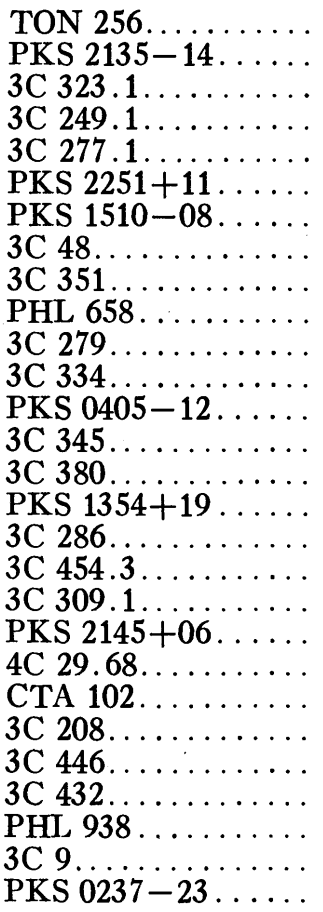 & $\begin{array}{l}0.1307 \\
0.202 \\
0.264 \\
0.311 \\
0.320 \\
0.323 \\
0.361 \\
0.367 \\
0.371 \\
0.450 \\
0.540 \\
0.556 \\
0.574 \\
0.595 \\
0.691 \\
0.720 \\
0.848 \\
0.859 \\
0.905 \\
0.990 \\
1.012 \\
1.037 \\
1.109 \\
1.403 \\
1.805 \\
1.930 \\
2.012 \\
2.202\end{array}$ & $\begin{array}{l}-0.97 \\
-0.75 \\
-0.92 \\
-0.46 \\
-0.28 \\
-0.98 \\
-1.13 \\
-1.38 \\
-0.66 \\
-1.51 \\
-0.45 \\
-0.25 \\
-1.50 \\
-0.38 \\
-0.31 \\
-0.22 \\
-1.49 \\
-0.23 \\
-0.13 \\
-0.35 \\
-0.95 \\
-1.50 \\
-1.58 \\
-1.13 \\
-0.80 \\
-1.50 \\
-0.95\end{array}$ & $\begin{array}{l}26.36 \\
26.91 \\
26.67 \\
27.13 \\
26.36 \\
27.08 \\
26.86 \\
26.90 \\
27.48 \\
26.76 \\
27.35 \\
27.0 \\
27.06 \\
27.33 \\
27.46 \\
27.32 \\
27.90 \\
27.49 \\
27.82 \\
27.42 \\
27.40 \\
27.44 \\
27.41 \\
27.79 \\
28.09 \\
27.88 \\
28.28\end{array}$ \\
\hline
\end{tabular}

* The redshifts for all objects except PKS $2145+06$ are taken from Burbidge (1967).

\section{OBSERVATIONAL RESULTS}

The quasi-stellar objects for which extensive observations have been obtained are listed in Table 1. The observed fluxes are plotted in Figures 1-8 as a function of the rest frequency $\nu_{0}=\nu(1+z)$. Standard deviations are shown for all measurements at $2.2 \mu$ and for all scanner measurements where the standard deviations in $\log f_{v}$ are greater than 0.02. The locations of some of the stronger emission lines are marked on each graph. There is no published redshift for PKS 2145+06. Two very weak lines at 3824 and $4682 \AA$ (Kinman and Burbidge 1967; Burbidge 1968) and a strong emission feature at $10000 \AA$ are identified respectively with $\lambda 1909$ of $\mathrm{C}$ III], $\lambda 2326$ of $\mathrm{C}$ II, and $\mathrm{H} \beta+[\mathrm{O} \mathrm{III}]$, and correspond to a redshift of 0.99 . In a few cases where blue spectrophotometric observations have still not been obtained, the blue end of the spectrum is represented only by 
the $U B V$ magnitudes converted to absolute fluxes; these points may be seriously influenced by strong emission lines. Wampler (1968) has obtained the energy distribution from 3300 to $9000 \AA$ of PKS 2251+11; his data form a smooth extension into the violet of the red results shown in Figure 2.

In addition to the measurements presented in Figures 1-8, data were obtained at $2.2 \mu$ on fifteen additional quasi-stellar objects; these are listed in Table 2. Except for PKS 1326+06 and PKS 2128-12, infrared measurements of all the quasi-stellar objects

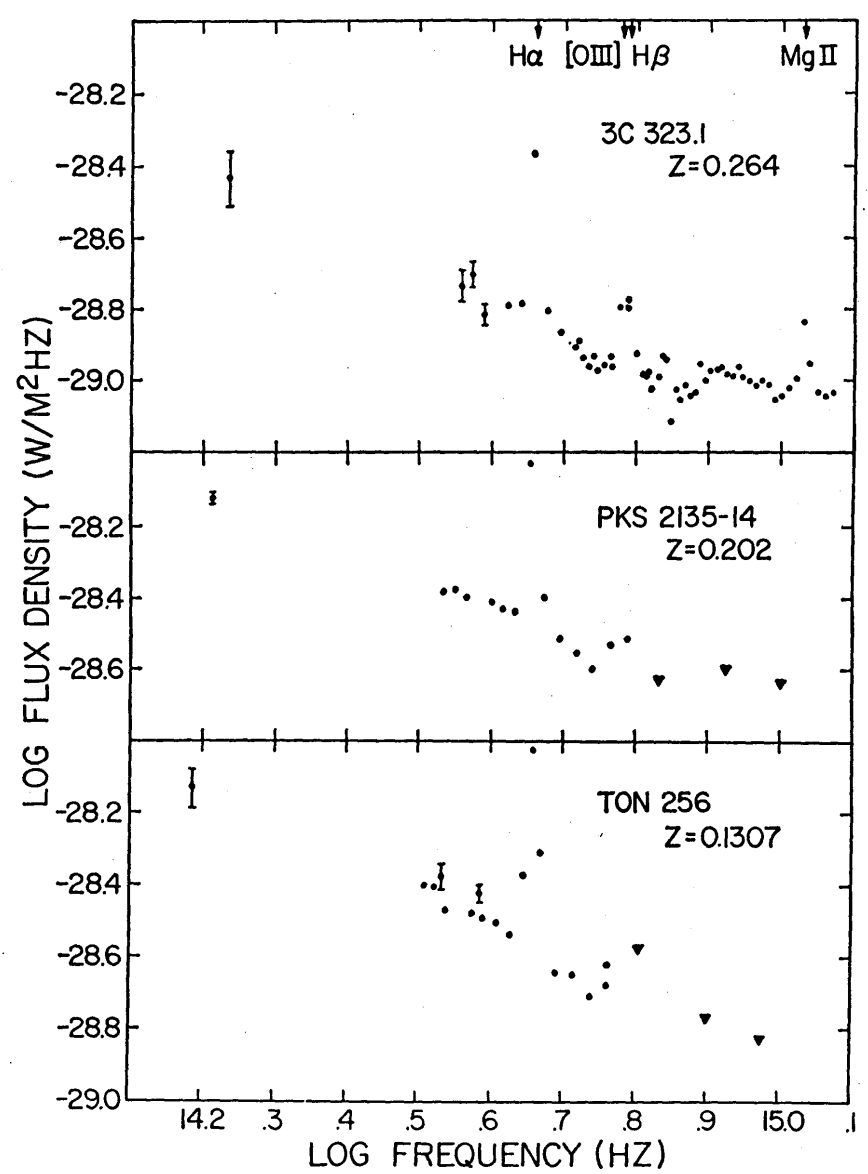

Fig. 1.-Observed flux plotted as a function of the rest frequency $\nu_{0}=\nu(1+z)$. Circles, data of this paper; inverted triangles, UBV measurements (Burbidge 1967). Redshifts are taken from Burbidge (1967). Standard-deviation error bars are shown for the infrared photometry and for all the scanner measurements where the standard deviation in $\log f_{\nu}$ is greater than 0.02 .

brighter than $m_{v}=16.8$ listed by Burbidge (1967) with right ascensions from $13^{\text {h }}$ to $5^{\text {h }}$ were attempted and are shown in either Figures 1-8 or Table 2.

Of the twenty-nine objects measured in the visual, eighteen were observed more than once. Although each measurement was limited to either the blue or the red, all measurements included a common point near $5500 \AA$. Only the four known variable objects 3C 279, 3C 345, 3C 446, and 3C 454.3 (Burbidge 1967) showed significant variation, and for these the individual data are shown. Of the forty-three quasi-stellar objects measured at $2.2 \mu$, twenty-one were measured more than once; eight of these were observed at times separated by more than 6 months so that some measure of the long-term variability could be made. Two sources, 3C 279 and 3C 345, showed statistically significant variations; unfortunately, observing conditions prevented 2.2- $\mu$ measurements of $3 \mathrm{C} 446$ and $3 \mathrm{C} 454.3$ from being repeated after a long time interval. 


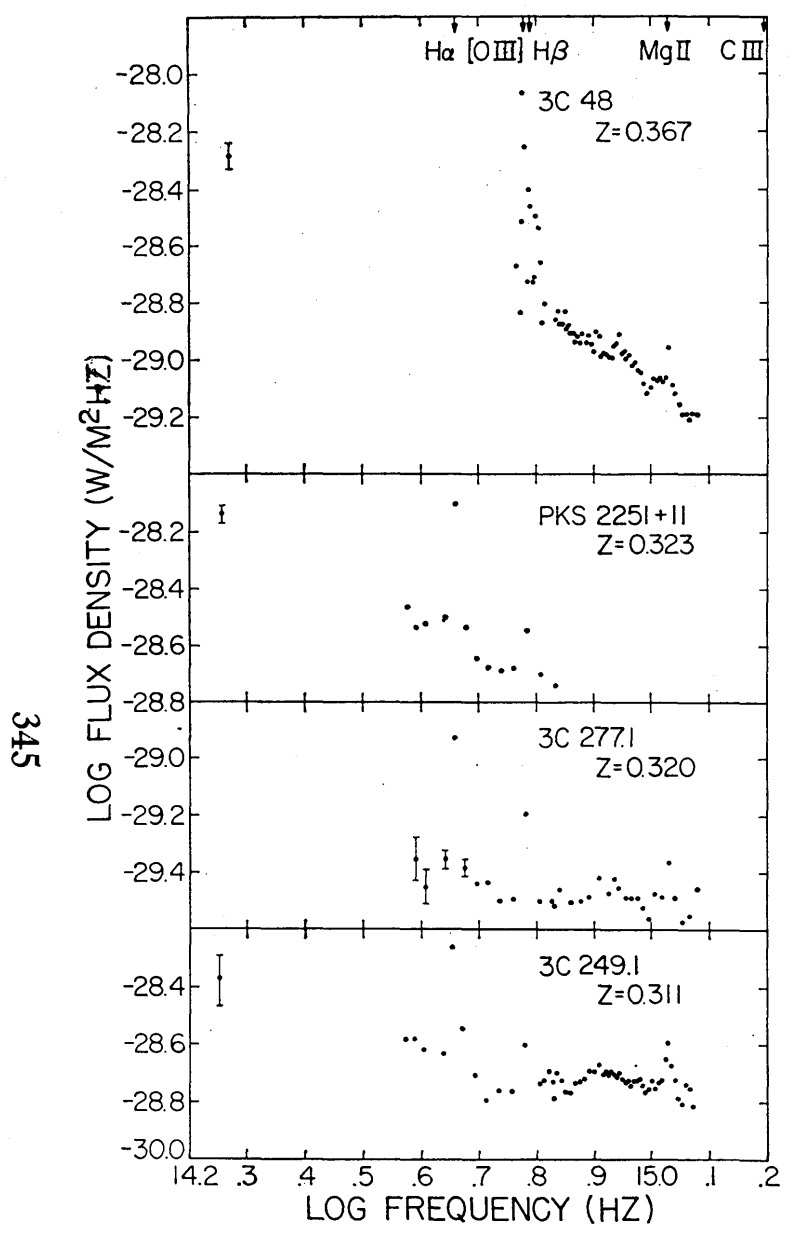

Fig. 2

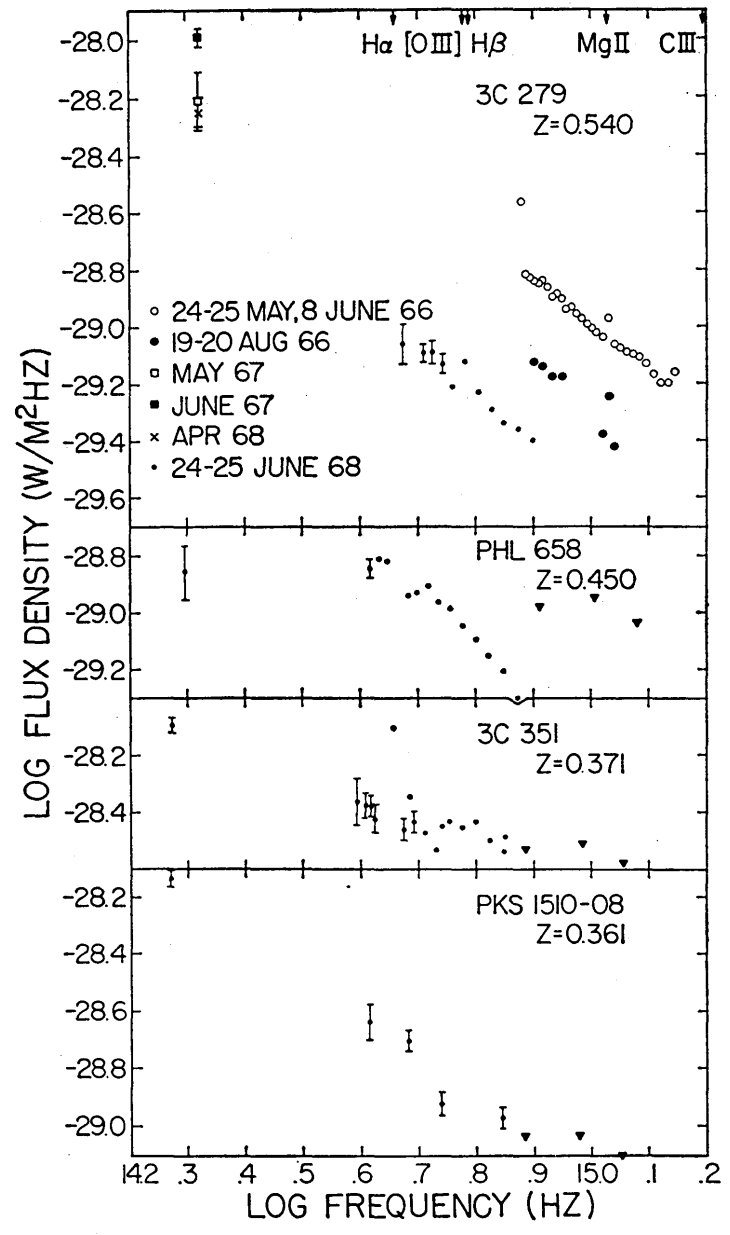

Fig. 3

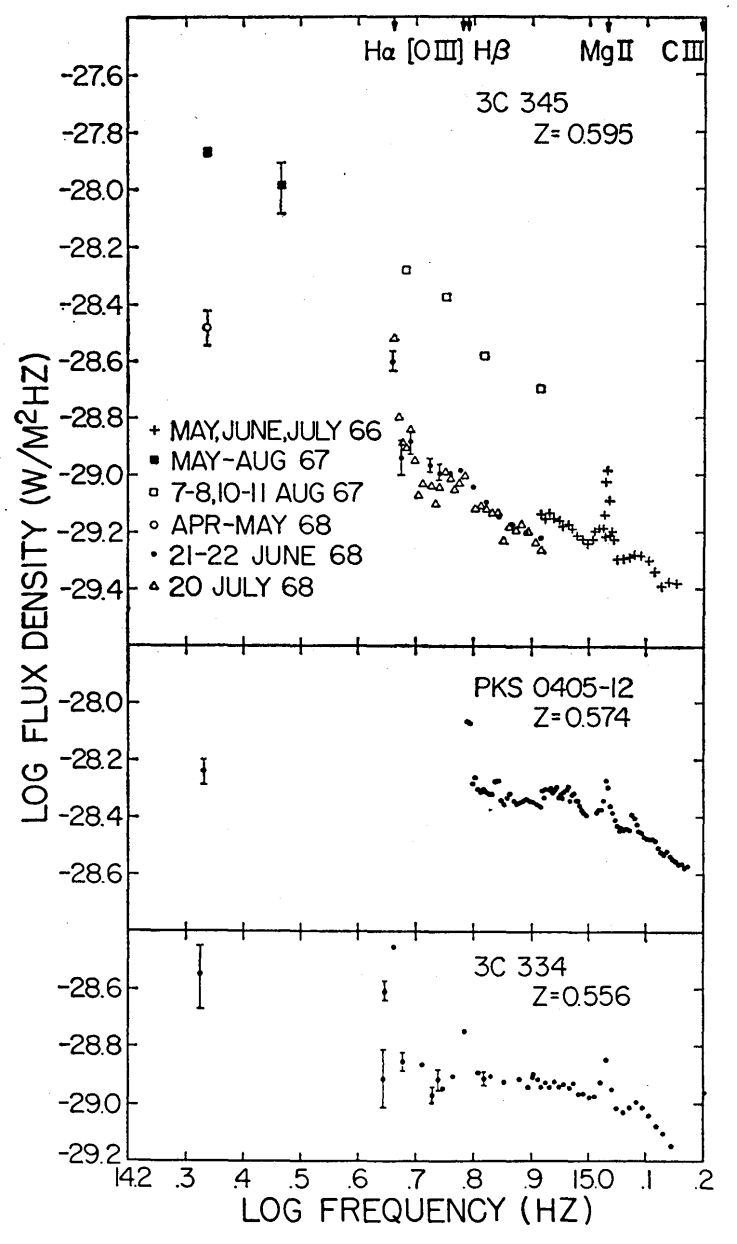

FIG. 4

Fig. 2.-Same as Fig. 1

Fig. 3.-Same as Fig. 1. For 3C 279 the measurements in both the visual and the infrared showed variability which is reflected in the special designations for each observation. The inconsistency between the scanner data and the $U B V$ for PHL 658 is unresolved and is probably instrumental.

Fic. 4.-Same as Fig. 1. For 3C 345 both the visual and infrared measurements showed variability as noted. 

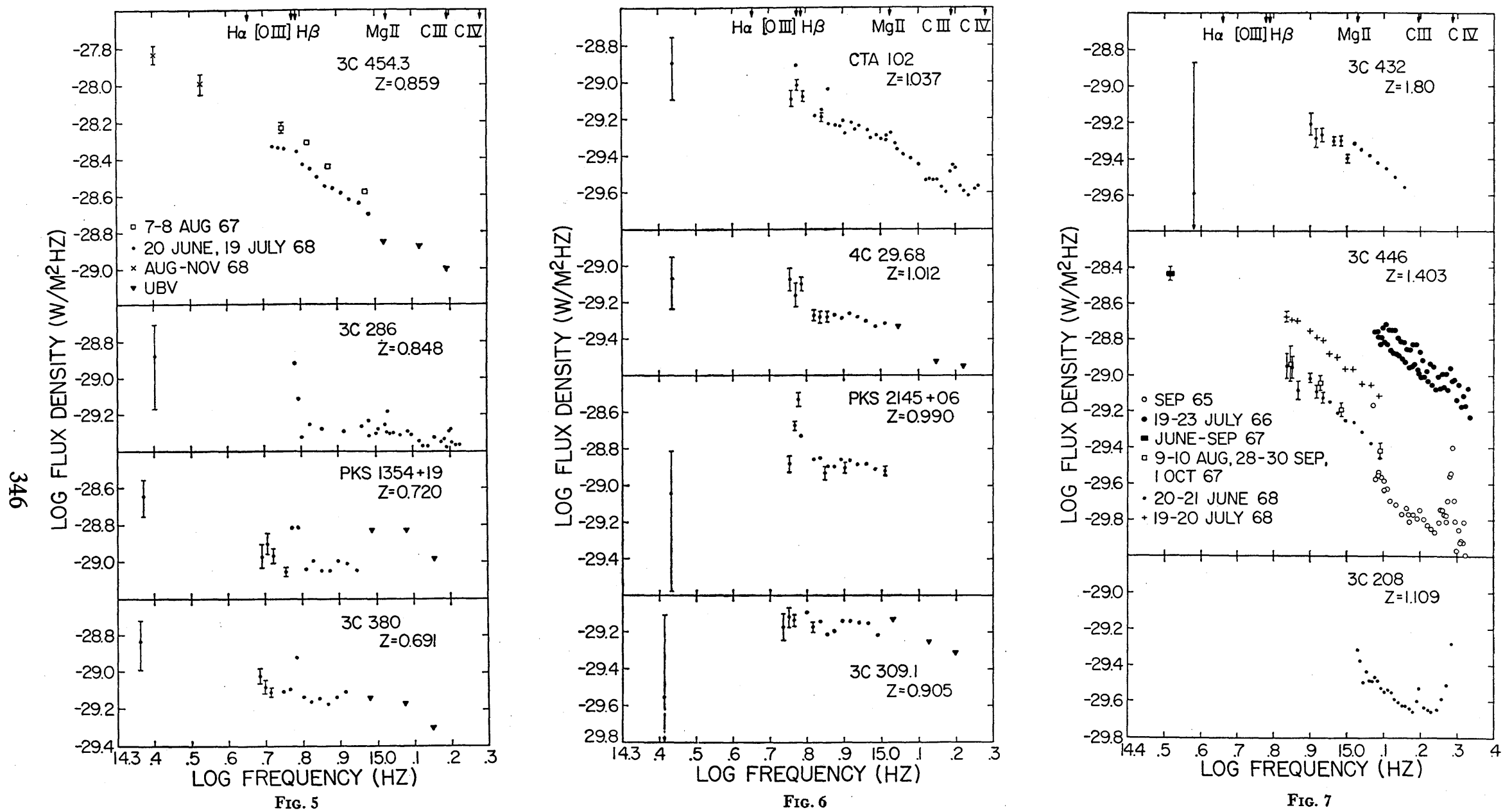

Fig. 5.-Same as Fig. 1. 3C 454.3 showed variations in the visual region; the infrared photometry was not repeated after a sufficient period of time to check for variability.

FIG. 6.-Same as Fig. 1. The $U B V$ measurements of 4C 29.68 are unpublished data of Sandage. The 2.2- $\mu$ point of 3C 309.1 is only a limit. The redshift of PKS 2145+06 is determined from these data and those of Kinman and Burbidge (1967) and Burbidge (1968).

Fig. 7.-Same as Fig. 1. The 2.2- $\mu$ point of 3C 432 represents only a limiting value. Visual variations were observed in 3C 446; the infrared photometry of 3C 446 was not repeated after a sufficient time period to check for infrared variability. 


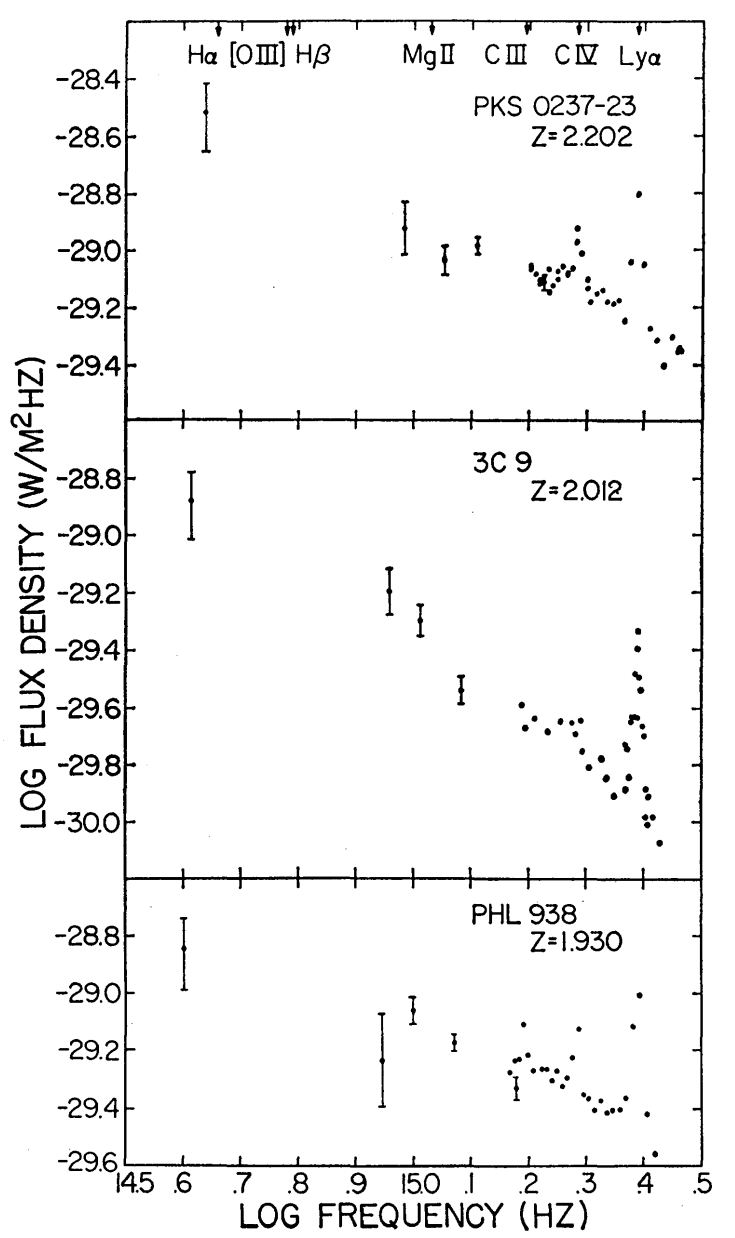

FIg. 8.-Same as Fig. 1

TABLE 2

2.2- $\mu$ MEASUREMENTS OF QUASI-STELLAR OBJECTS

\begin{tabular}{|c|c|c|c|}
\hline Object & $z^{*}$ & $\begin{array}{c}\log f_{\nu}(2.2 \mu) \\
\left(\mathrm{W} \mathrm{m} \mathrm{m}^{-2} \mathrm{~Hz}^{-1}\right)\end{array}$ & $a(V, 2.2 \mu) \dagger$ \\
\hline PKS $1327-21$. & 0.528 & $-28.68 \pm 0.20$ & -0.70 \\
\hline MSH 03-19. & 0.614 & $-28.46 \pm 0.08$ & -0.74 \\
\hline PKS 2344+09. & 0.667 & $-28.77 \pm 0.10$ & -0.03 \\
\hline $3 \mathrm{C} 57 \ldots \ldots \ldots$ & 0.68 & $-28.48 \pm 0.12$ & -0.81 \\
\hline $3 \mathrm{C} 175 \ldots$ & 0.768 & $-28.79 \pm 0.14$ & -0.44 \\
\hline $3 \mathrm{C} 336 \ldots$ & 0.927 & $-29.59 \pm 0.52$ & +0.04 \\
\hline MSH $14-121 \ldots$ & 0.940 & $-28.83 \pm 0.17$ & -0.87 \\
\hline 3C 287 . & 1.055 & $-29.16 \pm 0.31$ & -0.53 \\
\hline PKS 0122-00. & 1.070 & $-28.80 \pm 0.19$ & $(-0.03)$ \\
\hline PHL 1377. & 1.436 & $-29.05 \pm 0.13$ & +0.05 \\
\hline 3C 298. & 1.439 & $-28.89 \pm 0.16$ & -0.40 \\
\hline $4 C-4.8$ & 2.064 & $<-29.0$ & \\
\hline PKS 2154-18. & • & $-27.93 \pm 0.04$ & $(-1.77)$ \\
\hline PKS 2115-30. & & $-28.75 \pm 0.15$ & -0.41 \\
\hline PHL 1070. & & $-28.79 \pm 0.23$ & $(-1.07)$ \\
\hline
\end{tabular}

* The redshifts have been taken from Burbidge (1967).

$\dagger$ The visual magnitudes are taken from Burbidge (1967), except for 3C 175 which is unpublished data of Sandage. An $a(V, 2.2 \mu)$ in parentheses indicates that the Burbidge magnitude is uncertain. 


\section{DISCUSSION}

\section{a) Continuum}

It is clear from Figures 1-8 that a significant estimate of the location of the continuum spectrum can be determined provided it is assumed that all discrete features are emission lines. So that all the objects would be on a comparable basis, the flux $F_{\nu_{0}}$ in the continuum spectrum as emitted at the object has been estimated by using the value

$$
\dot{F}_{\nu_{0}}=1.07 \times 10^{57} f_{\nu_{0}} z^{2} /(1+z) ;
$$

estimates of this flux at a rest frequency $\nu_{0}=10^{15} \mathrm{~Hz}$ are included in Table 1. The above relationship depends on the Hubble constant (here taken as $100 \mathrm{~km} \mathrm{sec}^{-1} \mathrm{Mpc}^{-1}$ ) and the cosmological model (here assumed to correspond to $q_{0}=+1.0$ [Mattig 1958; Sandage 1961]). Had a model with $q_{0}=0$ been used, the fluxes would have been increased by a factor $\left(1+\frac{1}{2} z\right)^{2}$.

The flux $F_{\nu_{0}}$ at a rest frequency of $10^{15} \mathrm{~Hz}$ is also plotted against the redshift $z$ in Figure 9. The effects of the selection bias on this plot are shown by the solid curve, which

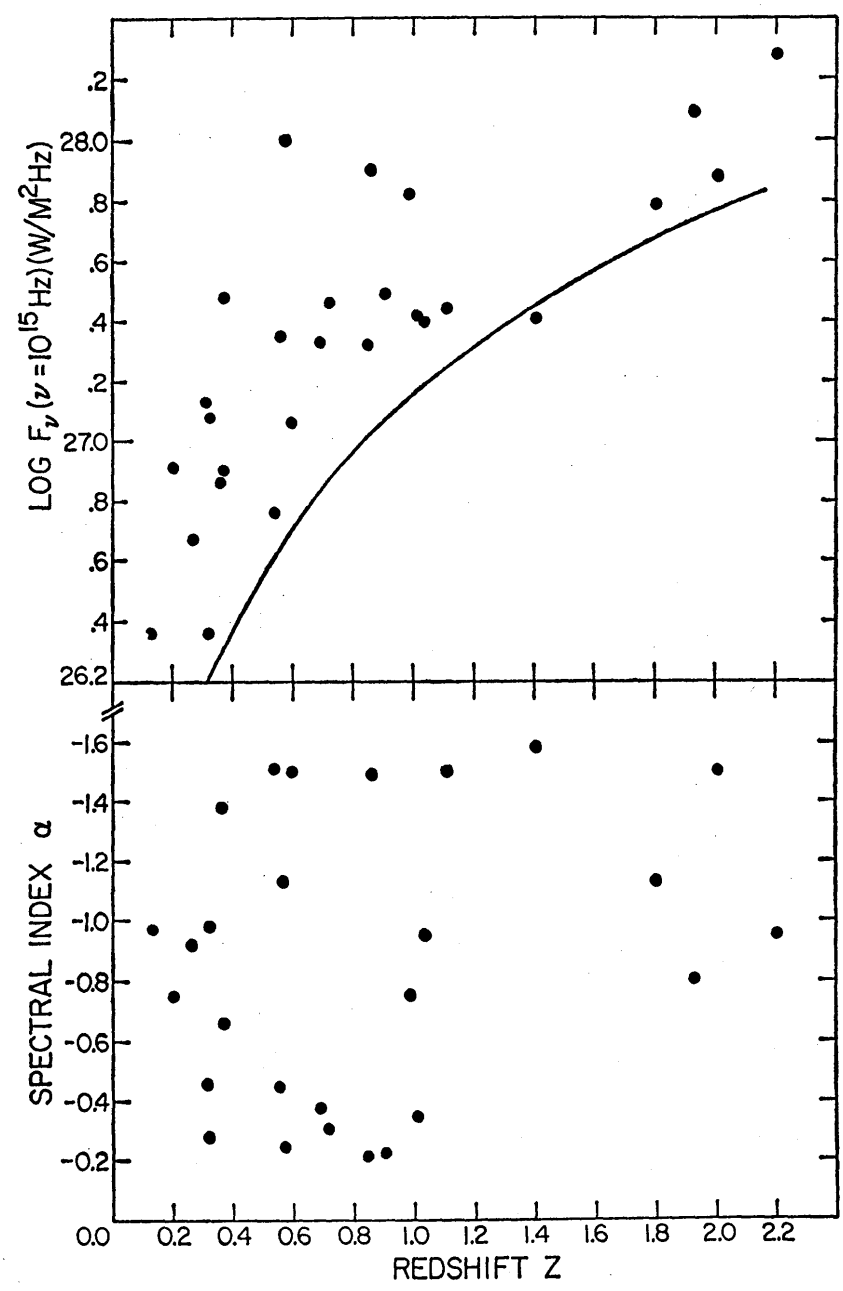

FIG. 9.-Estimates of the spectral index $a$ and of the flux $F_{\nu_{0}}$ of the continuum spectrum as emitted at the quasi-stellar object at a rest frequency of $10^{15} \mathrm{~Hz}$, plotted as a function of redshift $z$ (Burbidge 1967). The apparent concentrations in both plots can be accounted for by selection effects. Bottom plot shows that blue and bright quasi-stellar objects were preferentially studied with the spectrometer. In top plot, solid line delineates the limit for quasi-stellar objects with a visual magnitude of 18 ; a spectral index of -1 and $q_{0}=1.0$ are assumed. 
is an approximate lower limit derived by assuming that the faintest visual magnitude for which quasi-stellar sources have been observed is 18. The large scatter in Figure 9 clearly rules out deciding upon a cosmological model from these data.

Figures 1-8 show that generally a power-law representation of the continuum is valid. A spectral index $a$ defined by $f_{\nu} \propto \nu^{a}$ has been estimated for the objects shown, despite the fact that some continua clearly have curvature. An index for PHL 658 was not estimated because of the unresolved inconsistency between the scanner results and the $U B V$ photometry. As seen from Table 1 , the indices extend from -0.2 to -1.6 , with the whole range being populated. A plot of $a$ versus the redshift $z$ is also shown in Figure 9. The apparent concentration of low values of $a$ at low values of $z$ is not significant, since some of the objects observed with the spectrometer were chosen because of their brightness and relatively blue colors; there are known large-redshift cases which also have blue colors but which have not been studied. An estimate of the spectral index $a(V, 2.2 \mu)$ using the observed 2.2- $\mu$ flux and the $V$-magnitude listed by Burbidge (1967) has also been made for each of the fifteen objects tabulated in Table 2. These values, which are included in Table 2, are in agreement with the spectral indices estimated from Figures 1-8.

The radio-quiet objects observed-TON 256, PHL 938, and PHL 1070-appear to be indistinguishable from those with strong radio emission.

It has been noted that correlations exist between the $U B V$ colors of quasi-stellar sources and their redshifts (Barnes 1966; Kardashev and Komberg 1966; McCrea 1966). Sandage (1966) interpreted this correlation in terms of a universal absolute spectral energy distribution of the continuum with colors being produced by both the redshift and the emission lines. Strittmatter and Burbidge (1967), on the other hand, present arguments to show that the nonredshifted continuum is not unique and that the correlations with color are primarily a consequence of the various emission lines; a further detailed discussion is given by Lari and Setti (1967). The present data show that there is no unique nonredshifted continuum energy distribution. This is emphasized by the very large range in $a$ at all values of $z$ quoted above.

Because of the limitations in the completeness of the observing program, any conclusion with regard to the variability must be highly tentative. It has been noted by Peach (1969), among others, that, of the brighter quasi-stellar objects, only 3C 279, 3C 345, 3C 446, and 3C 454.3 show short-period optical variations with amplitudes greater than 1 mag. As can be seen from Figures 3, 4, 5, and 7, these amplitude changes occur with little obvious change in the shape of the energy distribution in the visual region. Furthermore, the data in Figures 3 and 4 show that, within the limitations of the lack of simultaneity, the infrared and visual continua of 3C 279 and 3C 345 follow each other. Thus, for these objects the same mechanism is probably responsible for the production of the radiation from the violet to the infrared.

Finally, it is perhaps not premature to point out that the four objects which exhibit large optical variations all also have energy distributions which rise steeply into the infrared. In fact, all four are among the seven objects with spectral indices near -1.5 .

It is instructive to see how the data presented here fit with those obtained in the radio wavelengths. In Figure 10 a summary of the trends exhibited in Figures 1-8 is presented, along with the available radio data. Sources TON 256 and PHL 938, which are radioquiet, are not included; $3 \mathrm{C} 273$ is added to provide a comparison with this well-known object. The figures must be viewed only as an indication of trends, since the ratio source and the optical source may not coincide.

Perhaps the most striking feature of Figure 10 is the absence of data between $10^{10}$ and $10^{14} \mathrm{~Hz}$; with few exceptions, there are no data published in this frequency range. Since a large fraction of the total energy could be emitted between these frequencies, any estimate of the total luminosity is extremely unreliable. Furthermore, in some cases the estimate would depend critically on whether the optical or the radio data are used to extrapolate into the far-infrared wavelengths. In particular, if the data between $2 \mu$ and 
$3 \mathrm{~cm}$ were missing for $3 \mathrm{C} 273$, the estimate of the total luminosity of that object could easily change by as much as a factor of 10 .

As is evident from both Figures 9 and 10, an extrapolation of the optical data does not, in general, lead to the conclusion that most of the energy is always radiated in the infrared. In fact, of the twenty-four objects selected as having their apparent $V$-magnitudes brighter than 16.8 and lying at right ascensions from $13^{\mathrm{h}}$ to $5^{\mathrm{h}}$, only five have an optical spectral index steeper than -1 and thus have more energy in the infrared than in the visual. Extrapolations of the radio data lead to the same conclusion.

One model of the radiation spectra of the quasi-stellar sources, which can be compared directly with the present data, is that in which the radiation in the optical continuum is a combination of the upper cutoff of a synchrotron spectrum which joins with, and is then dominated by, the inverse-Compton spectrum at some critical frequency. Takarada (1968) has applied such a model to Oke's previous data (Oke 1966, 1967). The present measurements are generally consistent with this interpretation. Thus, 3C 345 might

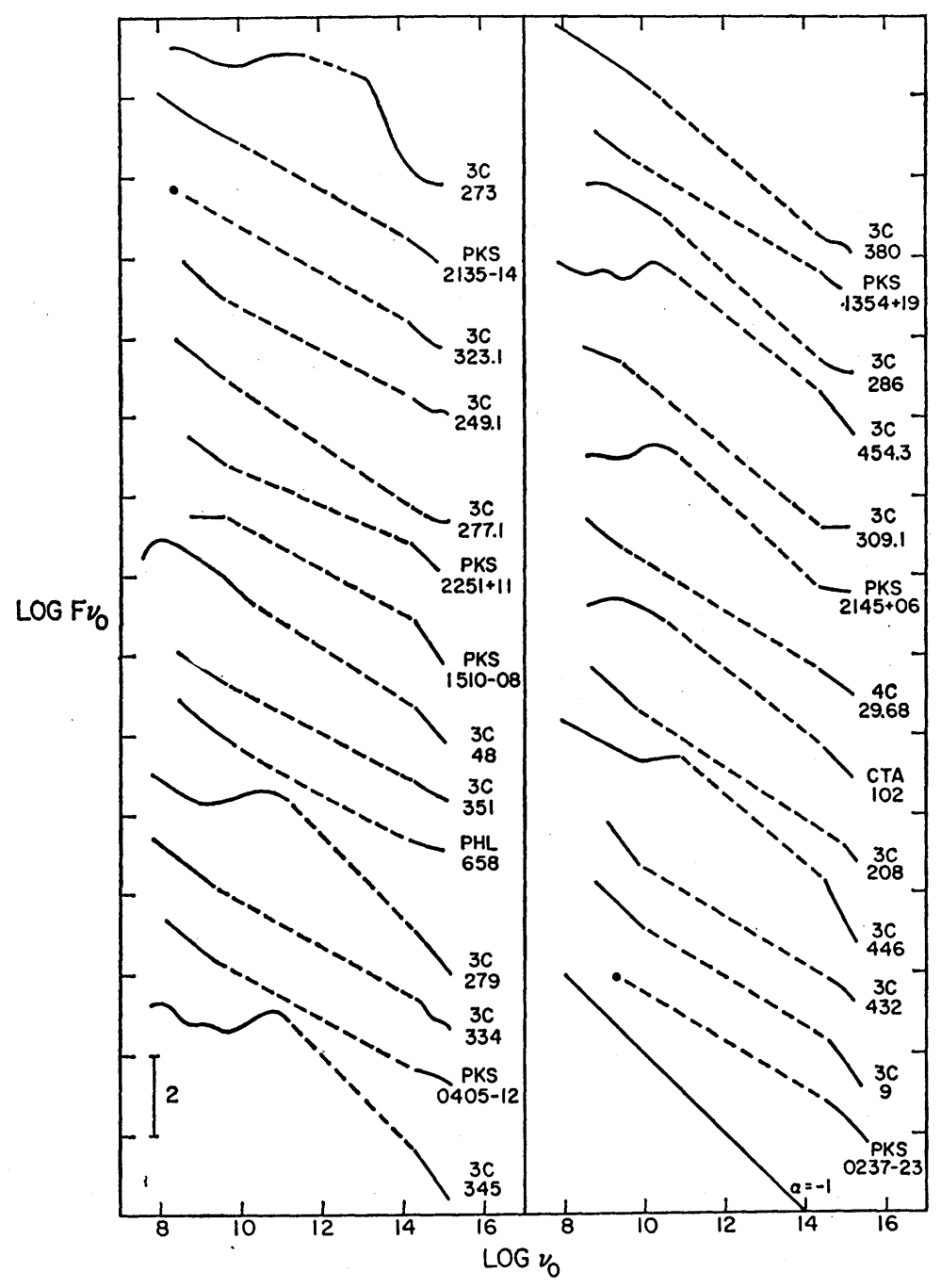

Fig. 10.-The trends of the data of Figs. 1-8 are summarized in order of redshift $z$, together with the radio data for the radio-active quasi-stellar sources. Dashed lines are only straight lines connecting the two types of data and are not meant to represent the actual spectra. The radio data are taken from Bennett (1962); Day et al. (1966); Shimmins et al. (1966); Arp, Bolton, and Kinman (1967); and Kellermann and Pauliny-Toth (1969). The data on 3C 273 are from Low (1968). 
show the synchrotron cutoff spectrum, 3C 309.1 would correspond to the inverseCompton spectrum, while 3C 249.1 would be typical of the region of the critical frequency. This picture must, of course, be a gross oversimplification. In particular, it does not account for the possibility that the radio and optical radiation may not be directly connected.

\section{b) Emission Lines}

In the course of making the spectrophotometric observations a special effort was made to obtain intensities of the stronger emission lines. In the blue spectral range, the bandpass was usually smaller than the emission-line breadths; thus, both the strength and width of the lines were obtained. In the red and near-infrared, the bandpass generally exceeded the line width; since the wavelengths for these multichannel observations were chosen to center the stronger lines within one of the channels, the total line intensity was obtained. There can remain a few cases where the line is so broad that even the $360 \AA$ bandpass used did not include the whole line.

Results of the emission-line data are summarized in Table 3. For each object, the equivalent widths $(W)$ and absolute emitted fluxes $(J)$ of the various lines found in that object are listed. The equivalent width (in angstroms) is defined in terms of the observed continuum; the observed flux in the line is given by

$$
j=3 \times 10^{10} f_{\nu}\left[W(\AA) / \lambda^{2}(\mu)\right],
$$

where $f_{\nu}$ is the continuum flux at the line. If a cosmological model with $q_{0}=+1$ and a Hubble constant of $100 \mathrm{~km} \mathrm{sec}^{-1} \mathrm{Mpc}^{-1}$ are assumed, the absolute emitted flux $J$ is given by

$$
J=1.07 \times 10^{57} z^{2} j
$$

In two cases, $\mathbf{H} \beta$ has been measured separately from the [O III] lines $\lambda \lambda 4959$ and 5007 ; in the remainder only the combined intensity is given. All $\mathrm{Ha}$ intensities include the $[\mathrm{N} \mathrm{Ir]} \mathrm{lines} \lambda \lambda 6548$ and 6583 if they are present.

A discussion of the ratio $\mathrm{Ha} / \mathrm{H} \beta$ in quasi-stellar sources is made difficult by the blending mentioned above. There is no possibility of separating the [N II] lines from $\mathrm{Ha}$ because of the small wavelength separation and great line widths. However, the importance of [N II] can perhaps be ascertained from measurements of the Seyfert galaxies. Anderson (1968) has studied eight Seyfert galaxies and finds that the [N II] lines contribute from 10 to nearly 50 percent of the emission-line strength near $\mathrm{Ha}$. The separation of [O III] and $\mathrm{H} \beta$, although possible, is generally not accomplished in the present data. In the two cases where [O III] and $\mathrm{H} \beta$ are separated, the ratio $[\mathrm{O} \mathrm{III}] / \mathrm{H} \beta$ is found to be 2.4 and 2.9. For 3C 249.1 and PKS 2251+11, Wampler (1968) has measured the $\mathrm{H} \beta$ and [O III] lines separately and finds ratios $[\mathrm{O} \mathrm{III}] / \mathrm{H} \beta$ of 1.95 and 0.7 , respectively. For the Seyfert galaxies, Anderson (1968) finds [O III] to be at least equal to $\mathrm{H} \beta$. If one assumes radiative recombination, the ratio $\mathrm{Ha} / \mathrm{H} \beta$ is expected to equal 2.75. The observed ratios $(\mathrm{H} a+[\mathrm{N} \mathrm{III}]) /(\mathrm{H} \beta+[\mathrm{O} \mathrm{III}])$ range from 2.3 to 5.6 ; in view of the potential contamination this ratio is consistent with an actual ratio $\mathrm{Ha} / \mathrm{H} \beta$ of 2.75 , and no significant departure from radiative recombination can be demonstrated. If, however, the [N II] lines are indeed weak, then Wampler's measures of 3C 249.1 and PKS $2251+11$ clearly indicate a large decrement and, in fact, the observations require that the [N II] strengths exceed that of $\mathrm{Ha}$ to be consistent with recombination theory.

One striking feature of Table 3 is the small range in the equivalent width of $\mathrm{Ha}$; the total spread is less than a factor of 3. A similar result has been found by Searle and Sargent (1968) for extragalactic objects which have broad emission lines. If the line is formed by radiative recombination, the ratio of the photon flux in $\mathrm{Ha}$ to the photon flux beyond the Lyman limit should be approximately 1:3. The number of photons emitted per second beyond the Lyman limit has been calculated on the basis of the esti- 
TABLE 3

OBSERVEd EMISSION-LiNe STRENGThS

\begin{tabular}{|c|c|c|c|c|c|c|c|c|c|c|c|c|}
\hline \multirow[b]{2}{*}{ Ов JЕСт } & \multicolumn{2}{|c|}{$\mathrm{Ha}, \lambda 6562$} & \multicolumn{2}{|c|}{$\mathrm{H} \beta+[\mathrm{O} \mathrm{Irr}], \lambda 4922$} & \multicolumn{2}{|c|}{ Mg Ir, $\lambda 2798$} & \multicolumn{2}{|c|}{ C IIr, $\lambda 1909$} & \multicolumn{2}{|c|}{$\mathrm{C}$ rv, $\lambda 1550$} & \multicolumn{2}{|c|}{$\mathrm{La}, \lambda 1216$} \\
\hline & $\begin{array}{l}W \\
(\AA)\end{array}$ & $\underset{(\text { ergs sec }}{\left.J \times 10^{-42}\right)}$ & $\begin{array}{r}W \\
(\AA)\end{array}$ & $\begin{array}{c}J \times 10^{-42} \\
\left(\operatorname{ergs~} \sec ^{-1}\right)\end{array}$ & $\begin{array}{l}W \\
(\AA)\end{array}$ & $\begin{array}{l}J \times 10^{-42} \\
\left(\text { ergs } \sec ^{-1}\right)\end{array}$ & $\begin{array}{l}W \\
(\AA)\end{array}$ & $\begin{array}{l}J \times 10^{-42} \\
\left(\text { ergs } \sec ^{-1}\right)\end{array}$ & $\begin{array}{l}W \\
(\AA)\end{array}$ & $\begin{array}{l}J \times 10^{-42} \\
\left(\text { ergs } \sec ^{-1}\right)\end{array}$ & $\begin{array}{l}W \\
(\AA)\end{array}$ & $\begin{array}{c}J \times 10^{-42} \\
(\text { ergs sec } \\
-1)\end{array}$ \\
\hline TON 256. & 572 & 15 & & & & $\cdots$ & . & $\cdots$ & $\ldots$ & $\cdots$ & . & $\cdots$ \\
\hline PKS $2135-14 \ldots \ldots$ & 748 & 51 & 133 & 12 & $\because$ & & $\cdots$ & $\cdots$ & $\cdots$ & $\cdots$ & $\ldots$ & $\ldots$ \\
\hline $3 \mathrm{C} 323.1 \ldots \ldots \ldots$ & 710 & 54 & 266 & 15 & 84 & 13 & $\ldots$ & $\ldots$ & $\ldots$ & $\ldots$ & $\ldots$ & $\ldots$ \\
\hline $3 \mathrm{C} 249.1 \ldots \ldots \ldots$ & 744 & 67 & 190 & 24 & 75 & 29 & $\ldots$ & $\ldots$ & $\ldots$ & $\ldots$ & $\ldots$ & $\ldots$ \\
\hline $3 \mathrm{C} 277.1 \ldots \ldots \ldots$ & 857 & 15 & 375 & 9.3 & 109 & 7.4 & $\ldots$ & $\ldots$ & $\ldots$ & $\ldots$ & $\ldots$ & $\ldots$ \\
\hline PKS $2251+11 \ldots \ldots$ & 928 & 102 & 198 & 30 & & & $\ldots$ & $\cdots$ & $\cdots$ & $\cdots$ & $\cdots$ & $\cdots$ \\
\hline $3 \mathrm{C} 48 \ldots \ldots \ldots \ldots$ & & 0 & $\int 110(\mathrm{H} \beta)$ & 17 & 15 & 3.3 & $\cdots$ & $\cdots$ & $\cdots$ & $\cdots$ & $\cdots$ & $\cdots$ \\
\hline $3 \mathrm{C} 351 \ldots$ & $\cdots$ & $\cdots$ & 264 (O III) & 40 & $\cdots$ & $\cdots$ & $\cdots$ & $\cdots$ & $\cdots$ & $\cdots$ & $\cdots$ & $\cdots$ \\
\hline $\begin{array}{l}3 \mathrm{C} 351 \ldots \ldots \ldots \ldots \ldots \\
3 \mathrm{C} 334 \ldots \ldots \ldots \ldots\end{array}$ & 375 & 76 & 88 & $\begin{array}{l}27 \\
33\end{array}$ & $\ddot{7 i}$ & $3 \ddot{3}$ & $\cdots$ & $\cdots$ & $\cdots$ & $\cdots$ & $\cdots$ & $\cdots$ \\
\hline PKS 0405-12 . . . & 803 & 108 & $\begin{array}{l}155 \\
133\end{array}$ & $\begin{array}{r}33 \\
110\end{array}$ & $\begin{array}{l}71 \\
57\end{array}$ & $\begin{array}{r}37 \\
119\end{array}$ & $\cdots$ & $\cdots$ & $\cdots$ & $\cdots$ & $\cdots$ & $\cdots$ \\
\hline $3 \mathrm{C} 345 \ldots \ldots \ldots \ldots$ & $\begin{array}{l}\cdots \\
\ldots\end{array}$ & $\cdots$ & $\begin{array}{r}100 \\
97\end{array}$ & 16 & 89 & 27 & $\ldots$ & $\cdots$ & $\cdots$ & $\cdots$ & $\cdots$ & $\cdots$ \\
\hline $3 \mathrm{C} 380$. & $\ldots$ & $\ldots$ & 245 & 40 & $\ldots$ & $\ldots$ & $\cdots$ & $\cdots$ & $\cdots$ & $\cdots$ & $\ldots$ & $\ldots$ \\
\hline PKS $1354+19 \ldots \ldots$ & $\ldots$ & $\ldots$ & 432 & 98 & $\ldots$ &,$\ldots$ & & $\ldots$ & $\ldots$ & $\ldots$ & $\ldots$ & $\ldots$ \\
\hline $3 C 286$ & & & $86(\mathrm{H} \beta)$ & 13 & 15 & 6 & 10 & 8 & $\ldots$ & $\ldots$ & $\ldots$ & $\ldots$ \\
\hline $3200 . . \cdots \cdots$ & $\cdots$ & $\cdots$ & $\{250$ (O III) & 36 & $\cdots$ & $\cdots$ & $\cdots$ & $\cdots$ & $\ldots$ & $\ldots$ & $\ldots$ & $\ldots$ \\
\hline PKS $2145+06 \ldots$ & $\ldots$ & $\cdots$ & 565 & 237 & & $\therefore$ & ? & & $\ldots$ & $\cdots$ & $\cdots$ & $\cdots$ \\
\hline CTA $102 \ldots \ldots \ldots$ & $\ldots$ & $\ldots$ & 201 & 50 & 112 & 50 & 32 & 48 & 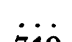 & & $\cdots$ & $\cdots$ \\
\hline $3 C 208 \ldots \ldots \ldots \ldots$ & $\ldots$ & $\cdots$ & $\ldots$ & $\ldots$ & $\ldots$ & $\ldots$ & 58 & 65 & 710 & 930 & $\ldots$ & $\ldots$ \\
\hline $3 \mathrm{C} 446 \ldots \ldots \ldots \ldots$ & $\ldots$ & $\ldots$ & $\ldots$ & $\ldots$ & $\ldots$ & $\ldots$ & 20 & 9 & 230 & 130 & & \\
\hline PHL $938 \ldots \ldots \ldots \ldots$ & $\ldots$ & $\ldots$ & $\ldots$ & $\ldots$ & $\ldots$ & $\ldots$ & 32 & 72 & 127 & 320 & 428 & 1270 \\
\hline $3 \mathrm{C} 9 \ldots \ldots \ldots \ldots$ & $\ldots$ & $\ldots$ & $\ldots$ & $\ldots$ & $\ldots$ & $\ldots$ & $\ldots$ & $\ldots$ & 98 & 100 & 460 & 500 \\
\hline PKS $0237-23 \ldots \ldots$ & $\ldots$ & $\ldots$ & $\ldots$ & $\ldots$ & $\ldots$ & $\ldots$ & $\ldots$ & $\ldots$ & 116 & 320 & 284 & 1670 \\
\hline
\end{tabular}


mated flux at $\nu_{0}=10^{15} \mathrm{~Hz}$ and the spectral index $a$ in the power-law fit of the continuum. Both this rate and the number of photons per second being emitted in $\mathrm{Ha}$ are shown in Table 4. While in four objects the ratios are consistent with the theoretical relation, in four other objects the extrapolated ultraviolet continuum is too strong by a factor of approximately 4 . In view of the large extrapolation of the continuum into the ultraviolet, however, one cannot conclude that the $\mathrm{Ha}$ intensities are inappropriate for radiative recombination.

The range in the equivalent width of the $\mathrm{L} a$ line is also small. For radiative recombination, when the emitting region is opaque to $\mathrm{L} a$, there should be one La photon for each photon beyond the Lyman limit, although at least a small amount of Lyman radiation escapes from the outer parts of the source where the optical depth is small. The number of photons per second in the continuum has been extrapolated by using the power-lawfitted spectrum, and the results are included in Table 4. The Lyman continuum appears to be too strong by a factor of 3 to 10 . Although the extrapolation can be quite uncertain and can perhaps account for a discrepancy by a factor of 3 , the discrepancy by a

TABLE 4

Calculated Number of Photons at Ha ANd L $a$, AND IN THE LYMAN CONTINUUM

\begin{tabular}{|c|c|c|c|}
\hline Object & $N(\mathrm{Ha} a) \times 10^{-55}$ & $N($ Ly continuum $) \times 10^{-55}$ & $N(\mathrm{~L} a) \times 10^{-6 b}$ \\
\hline $\begin{array}{l}\text { TON } 256 \ldots \ldots \ldots \\
\text { PKS } 2135-14 \ldots \ldots \ldots \\
\text { 3C } 323.1 \ldots \ldots \ldots \ldots \\
\text { 3C } 249.1 \ldots \ldots \ldots \ldots \\
\text { 3C } 277.1 \ldots \ldots \ldots \ldots \\
\text { PKS } 2251+11 \ldots \ldots \ldots \\
\text { 3C } 351 \ldots \ldots \ldots \ldots \ldots \\
\text { 3C } 334 \ldots \ldots \ldots \ldots \\
\text { PHL } 938 \ldots \ldots \ldots \ldots \\
\text { 3C } 9 \ldots \ldots \ldots \\
\text { PKS } 0237-23 \ldots \ldots \ldots\end{array}$ & $\begin{array}{l}0.50 \\
1.69 \\
1.79 \\
2.22 \\
0.50 \\
3.38 \\
2.52 \\
3.85 \\
\cdots \\
\cdots \\
\cdots\end{array}$ & $\begin{array}{r}1.2 \\
6.6 \\
2.6 \\
25.7 \\
8.7 \\
5.8 \\
31.6 \\
44.6 \\
91.0 \\
12.5 \\
98.0\end{array}$ & $\begin{array}{r}\cdots \\
\cdots \\
\cdots \\
\cdots \\
\cdots \\
\cdots \\
7.8 \\
3.1 \\
10.2\end{array}$ \\
\hline
\end{tabular}

factor of 10 present in two objects requires an extreme change in the continuum extrapolation, which in this case is a short distance in wavelength. There are at least two possible reasons for this discrepancy. (1) La can be destroyed by two-photon emission. The observed continuum energy in the neighborhood of $2400 \AA$, however, appears to rule out a two-photon flux several times larger than that in La. (2) The object may not be opaque to Lyman radiation. It is possible, for instance, that a model where the gas is concentrated in blobs could let a large fraction of the radiation in the Lyman continuum escape.

A further striking result of Table 3 is the almost constant ratio of the line intensities of $\mathrm{L} a$ to the $C$ IV line at $\lambda 1550$. The ratio of the line intensities can be easily calculated if one assumes that $\mathrm{L} a$ is formed by recombination in an optically thick source while $\lambda 1550$ is produced by collisional excitation (Oke 1966). The ratio depends strongly on the ratio of protons to $\mathrm{C}^{+++}$ions and on the electron temperature. If the hydrogen-tocarbon abundance ratio is the same as in the Sun and if all carbon is in the form of $\mathrm{C}^{+++}$, the observed ratio requires an effective temperature $T_{e}=20000^{\circ} \mathrm{K}$, with temperatures below $14000^{\circ}$ and above $30000^{\circ} \mathrm{K}$ being completely ruled out. The constancy of the ratio could also be realized if the $\mathrm{C}$ IV line is produced by the radiation field, either by radiative excitation to high levels followed by a cascade to the ground level or by ionization followed by recombination. The recombination process is unlikely, since it requires approximately equal abundances of protons and $\mathrm{C}^{++++}$ions. Radiative excitation also is unlikely, since higher-level C IV lines have not been observed. 
Both the $\lambda 1909$ line of $\mathrm{C} \mathrm{III}]$ and the $\lambda 2798$ line of $\mathrm{Mg}$ II are also probably produced collisionally. Since $T_{e}$ appears to have a small range, the large range in intensity observed for these lines is presumably caused by the different degrees of ionization in different objects and within the objects themselves.

A study of the most accurately observed objects shown in Figures 1-8 reveals several interesting spectral features. One region of interest is that between rest wavelengths 3200 and $4000 \AA$; this is shown for five objects in Figure 11. In four of these objects there is clearly complex emission. This has already been observed in 3C 48 (Oke 1966). Some of the emission can presumably be attributed to broad, weak forbidden lines$\lambda 3727$ of [O II], $\lambda \lambda 3868$ and 3967 of [Ne III], and $\lambda \lambda 3345$ and 342.5 of [Ne v]. The emission, however, sometimes extends below $\lambda 3200$. Identification with He II $\lambda 3202$ is unlikely,

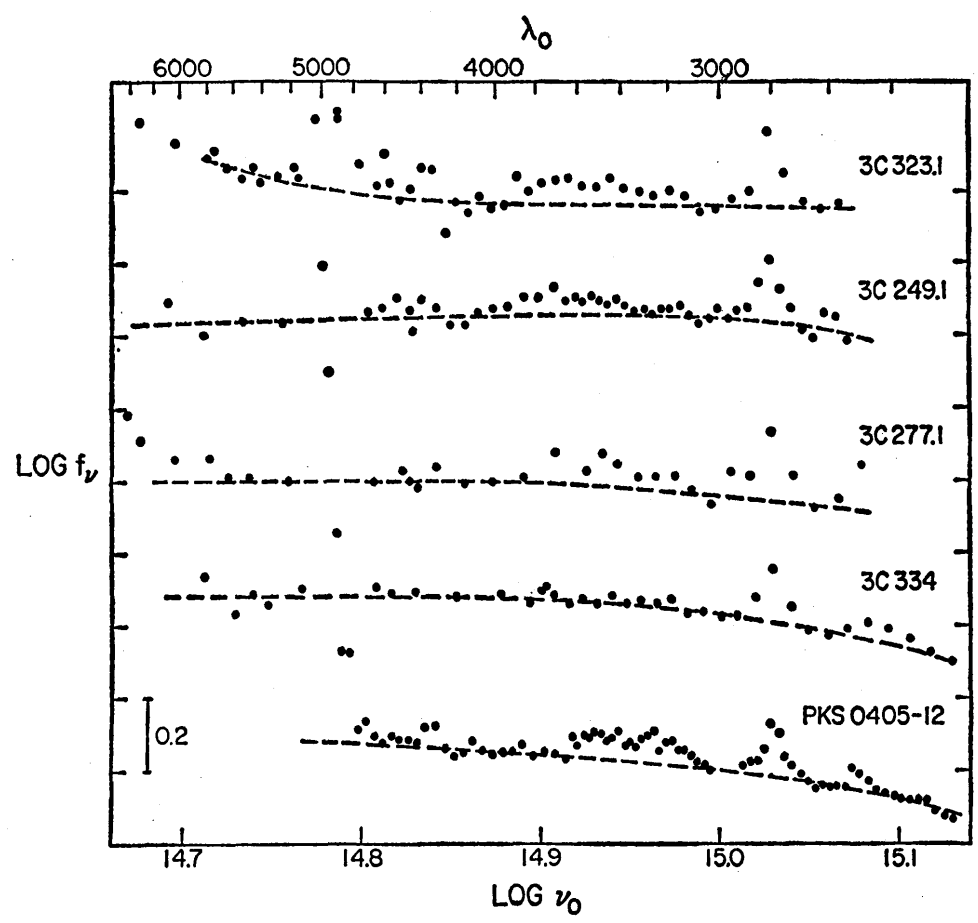

Fig. 11.-Spectral energy in a limited wavelength region, shown for five of the most accurately observed objects. Dashed curves, eye-fit estimate of the continuum radiation. The complex emission between $\lambda \lambda 3200$ and 4000 is clearly evident in 3C 323.1, 3C 249.1, 3C 277.1 and PKS 0405-12. 3C 334 is included to provide a comparison where this structure is minimal.

since the preceding line in the series at $\lambda 4686$ is either weak or absent. Another possible identification is with the fluorescent lines of $\mathrm{O}$ III, near $\lambda 3420$. These lines, however, should be accompanied by other lines near $\lambda 3040$ which are clearly absent. A further possibility is that a small Balmer jump occurs; this does not, however, seem to be the case in 3C 334.

An emission line at $\lambda_{0} \approx 2500$ is clearly present in PKS $0405-12$. It also appears somewhat weaker in 3C 334, 3C 345, and 3C 286. Possible identifications are with [Mg VII] or [O II].

\section{SUMMARY}

The data presented in this paper may be summarized as follows:

a) The energy distribution of the quasi-stellar sources from $3200 \AA$ in the violet through $2.2 \mu$ in the infrared can be described generally with a power-law spectrum.

b) The flux per unit frequency interval generally increases toward the infrared. Spectral indices are in the range -0.2 to -1.6 . 
c) The data to $2.2 \mu$ do not cover a sufficient fraction of the total spectrum to make accurate estimates of the total luminosity.

d) The energy distributions of the four known large-amplitude variables remain sensibly the same at different phases in the variation.

$e)$ No characteristic of the energy distribution in the wavelength range observed which distinguishes between radio-quiet and radio-active quasi-stellar objects is found.

$f$ ) The ratio of the line intensities of $\mathrm{L} a$ to the $\mathrm{C}$ IV line at $\lambda 1550$ is almost constant. If $\mathrm{L} a$ is formed by recombination and $\lambda 1550$ by collisional excitation, this defines an effective temperature of $20000^{\circ}\left(+10000^{\circ},-7000^{\circ}\right) \mathrm{K}$.

g) The range of equivalent width of both $(\mathrm{Ha}+[\mathrm{N} \mathrm{II}])$ and $\mathrm{L} a$ is extremely small. The data cannot be used to rule out the possibility that the radiation is caused by radiative recombination.

h) There are more photons in the Lyman continuum with respect to the number in the $\mathrm{L} a$ line than is consistent with radiative recombination from an opaque source.

We thank Gary Tuton, Judy Bennett, Kwong Chu, and Linda Murphy for help in obtaining, reducing, and presenting these data. We also thank Allan Sandage for permitting us to use his data before publication, Frank Low for discussing his data with us, and Robert Leighton and Gordon Garmire for reading and discussing the paper.

\section{REFERENCES}

Anderson, K. S. 1968, unpublished thesis, California Institute of Technology.

Arp, H. C., Bolton, J. G., and Kinman, T. D. 1967, Ap. J., 147, 840.

Barnes, R. C. 1966, Ap.J., 146, 285.

Becklin, E., and Neugebauer, G. 1968, Ap.J., 151, 145.

Bennett, A. S. 1962, Mem.R.A.S., 68, 163.

Burbidge, E. M. 1967, Ann. Rev. Astr. and Ap., 5, 399.

1968, Ap.J. (Letters), 154, L109.

Day, G. A., Shimmins, A. J., Ekers, R. D., and Cole, D. J. 1966, A ustralian J. Phys., 19, 35.

Johnson, H. L. 1962, Ap. J., 135, 69.

- 1964, ibid., 139, 1022 .

Johnson, H. L., Mitchell, R. I., Iriarte, B., and Wisniewski, W. Z. 1966, Comm. Lunar and Planet. Lab., 4, 99.

Kardashev, N. S., and Komberg, B. V. 1966, Astr. Cir. U.S.S.R., No. 357, p. 1.

Kellermann, K. I., and Pauliny-Toth, I. I. K. 1969, A p. J. (Letters), 155, L71.

Kinman, T. D., and Burbidge, E. M. 1967, Ap. J. (Letters), 148, L59.

Lari, C., and Setti, G. 1967, Nuovo Cimento, 52 (No. 2), 507.

Low, F. L. 1968, in Highlights of Astronomy, ed. L. Perek (New York: Springer Verlag), p. 136.

Low, F. L., and Johnson, H. L. 1965, Ap.J., 141, 336.

McCrea, W. H. 1966, Pub. A.S.P., 78, 49.

Mattig, W. 1958, Astr. Nach., 284, 109.

Mihalas, D. 1966, Ap. J. Suppl., 13, 1.

Oke, J. B. 1964, Ap. J., 140, 689.

… 1965a, ibid., 141, 6.

—. 1965b, Ann. Rev. Astr. and Ap., 3, 23.

- 1966, A p. J., 145, 668.

-. 1967, ibid., 147, 901.

- 1969, Pub. A.S.P., 81, 11.

Peach, J. V. 1969, Nature, 222, 439

Sandage, A. 1961, Ap.J., 133, 355. .1966, ibid., 146, 13.

Searle, L., and Sargent, W. L. W. 1968, Ap. J., 153, 1003.

Shimmins, A. J., Day, G. A., Ekers, R. D., and Cole, D. J. 1966, A ustralian J. Phys., $19,837$.

Strittmatter, P. A., and Burbidge, G. R. 1967, Ap. J., 147, 13.

Takarada, K. 1968, Progr. Theoret. Phys., 40, 770.

Wampler, E. J. $1967 a, A$ p. J., 147, 1.

. 1967b, Ap.J. (Letters), 148, L101.

- 1968, Ap. J., 153, 19.

Wampler, E. J., and Oke, J. B. 1967, Ap. J., 148, 695. 
